\title{
Efficacy of 'Thriposha' supplementation in improving the micronutrient status of preschool children
}

\author{
M Hettiarachchi $^{1}$, C Liyanage $^{2}$ \\ (Index words: preschool children, 'Thriposha' supplementation, anaemia, micronutrients)
}

\begin{abstract}
Introduction The islandwide comprehensive supplementary feeding scheme, the 'Thriposha' programme has been in existence in the country for over three decades. However, its effectiveness in improving micronutrient status has not yet been evaluated.

Objective We examined the effects of 'Thriposha' on micronutrient status, assessed haematologically and biochemically, in young children.

Methods Preschool children (aged 3-5 years) from two well-baby clinics were grouped into interventional $(n=137)$ arm and control $(n=130)$ arm. Children in the interventional group were fed 'Thriposha' ( $50 \mathrm{~g} /$ day) while the control group of children was fed with $50 \mathrm{~g}$ 'Thriposha' made without mineral and vitamin premix, for a period of nine months. Serum calcium, ferritin, folate, freeT4, ceruloplasmin, zinc, vitamin A, vitamin D and haemoglobin $(\mathrm{Hb})$ levels were measured before and one week after completing intervention.

Results The baseline $\mathrm{Hb}$ levels of the intervention and control groups were 113.20 (SD10.9) g/l and 112.30 (SD 9.0) $\mathrm{g} / \mathrm{l}$ respectively. After the intervention, the interventional group showed a significant improvement (repeated measures ANOVA, $p=0.02$ ) in the $\mathrm{Hb}$ (mean of 118.10 (7.7) vs. 114.70 (7.0) $\mathrm{g} / \mathrm{l}$ of the control). The prevalence of anaemia dropped from $37 \%$ to $15 \%$ in the intervention group ( $p=0.03)$. Serum ferritin and ceruloplasmin levels also improved.
\end{abstract}

Conclusion Regular consumption of conventional 'Thriposha' for 9 months led to improvements in $\mathrm{Hb}$, ferritin and ceruloplasmin levels in the blood.

Ceylon Medical Journal 2010; 55: 85-9

\section{Introduction}

Strategies used for combating micronutrient deficiencies include high-dose supplementation, food fortification, nutrition education, and food diversification [1]. Of these, the food-based approach is probably the most sustainable and desirable approach. However, changing the dietary habits of a population is not easy and may take years to show an effect. Food fortification, on the other hand, offers a solution that gives quicker results, and simultaneously reaches large segments of the population.
The island-wide comprehensive supplementary feeding scheme, known as the 'Thriposha' programme has been in existence in the country for over three decades, but its effectiveness in improving overall nutrtional status has not been evaluated so far. Therefore, we undertook an intervention trial in Southern Sri Lanka to determine the efficacy of Thriposha' supplementation in relation to growth, micronutrient status, bone health and cognitive abilities. Of these, the efficacy of 'Thriposha' in improving the micronutrient status of preschool children is reported in this paper.

\section{Methods}

The study protocol received approval from the Ethical Review Committee of the Faculty of Medicine, University of Ruhuna and the intervention study was registered in the Sri Lanka Clinical Trial Registry (SLCTR/ 2007/006) managed by Sri Lanka Medical Association. Two out of four public health clinics in this University field training area (Bope-Poddala Health Division) were randomly selected to facilitate regular monitoring over the study period without incurring high transportation costs. The two selected clinics were $10 \mathrm{~km}$ apart. Each clinic had three public health midwives (PHM) to serve the area. A list of children aged 3-5 years was obtained from the respective PHM and using a random number table, participants were selected for the study [2]. Next, parents of the study participants were invited to a meeting at which the purpose of the study, risks and benefits were explained. Informed consent was obtained from those families who agreed to participate.

Children who had any known chronic medical conditions, took any medications, or were consuming vitamin or mineral preparations in the preceding month were not included in the study. Two weeks before starting the study, each child was given $100 \mathrm{mg}$ of mebendazole twice daily for three days to eliminate worms. The children were subjected to a brief physical examination, their weights and heights were measured using a beam balance and stadiometer respectively. A venous blood sample (5 $\mathrm{ml}$ ) was obtained from each subject using disposable syringes and $20.0 \mu \mathrm{l}$ of whole blood was pipetted onto a

${ }^{1}$ Nuclear Medicine Unit and ${ }^{2}$ Department of Community Medicine, Faculty of Medicine, University of Ruhuna, Sri Lanka.

Correspondence: $\mathrm{MH}$, e-mail <nmu_galle@yahoo.com>. Received 11 December 2009 and revised version accepted 5 April 2010. Competing interests: none declared. 
filter paper for haemoglobin (Hb) measurement. The rest of the blood was collected in an acid washed centrifuge tube, serum was separated by centrifugation and stored at $-80^{\circ} \mathrm{C}$ until analysis for calcium, ferritin, folate, free $\mathrm{T} 4$, ceruloplasmin, zinc, vitamin A and vitamin D levels. The same measurements were carried out one week after the nine months of intervention.

The study sample was divided into intervention and control groups depending on the locality, after matching their initial age and anthropometry to make sure that both groups had similar characteristics at baseline. 'Thriposha' was provided to all children below 10 years of age in the household to make sure that the study participants would get the recommended daily portion (50g). The children in the intervention group were given conventional 'Thriposha' and the control group was fed with 'Thriposha' made without mineral and vitamin premix (Corn-Soya-BlendCSB) for a period of nine months. 'Thriposha' was given to each child daily preferably two hours after either breakfast or lunch. Conventional 'Thriposha' and CSB were ordered separately from the manufacturing plant in Kapuwatta, Ja-Ela, Sri Lanka and there were no differences in the packing material or size of the packet. The contents were blinded to the family. Distribution of 'Thriposha' was done at monthly intervals and was handled by the research team at the respective clinic. Compliance and consumption of this meal was checked periodically, with the help of public health midwife of the relevant clinic. Further, each family unit was asked to submit the remaining portion of their 'Thriposha' to get the new batch and to avoid excessive consumption by the child.

Laboratory analysis: Haemoglobin concentration was measured using the photometric cyanmethae-moglobin method at the Nutrition Research Laboratory of the Faculty of Medicine. Serum ferritin (SF) free thyroxine (fT4), ceruloplasmin, folate, vitamin D levels were measured by the immunoradiometric assay techniques at the Radioimmunoassay (RIA) laboratory of the Nuclear Medicine Unit, Faculty of Medicine, Galle using reagent kits of NETRIA, London. Serum calcium concentration was measured by the enzyme linked immunosorbant assay (ELISA) kit at the RIA laboratory using reagents supplied by Biodex USA. The serum retinol levels were determined by high performance liquid chromatography (HPLC) method and serum zinc by flame atomic absorption spectrometry (f-AAS) at the Industrial Technology Institute (ITI), Colombo. Anaemia was defined as hemoglobin concentration $<11.0 \mathrm{~g} /$, depleted iron stores as SF $<12.0 \mu \mathrm{g} / \mathrm{l}$, marginal iron stores as SF 12.1-30.0 $\mu \mathrm{g} / \mathrm{l}$ and iron deficiency anaemia (IDA) as anaemia with depleted iron stores [2].

\section{Statistical analysis}

The number of participants for the study was calculated as 80 per group with the assumption that the prevalence of anaemia in children would be $20 \%$ at $90 \%$ power ( $\alpha=0.05$ ). With possible $20 \%$ dropout rate during the period of intervention (i.e., 9 months), another 20-25 children were added to each group.
Differences between groups in the concentration of biochemical parameters at initial and post-intervention stages were investigated using multivariate analysis of variance (MANOVA) repeated-measures design with supplement type as a between-subject factor (two groups) and treatment effect (baseline compared with final) as a within-subject factor. Baseline values were also included in the analysis as between-subject factors to correct for their possible confounding influence on the change in respective variables being different at baseline.

\section{Results}

There were 137 participants in the intervention group and 130 participants in the control group at baseline. At the end of 9 months of intervention 33 children defaulted from the intervention group and 31 from the control resulting an overall drop-out rate of $24.0 \%$. As such, a total of 104 children in the intervention group and 99 children in the control group completed the trial. The mean (SD) levels of $\mathrm{Hb}$ and other serum parameters are presented in Table 1. The baseline Hb level of the intervention and control groups were 113.20 (10.9) g/l and 112.30 (9.0) g/l respectively. As a result of supplementation, the intervention group showed a significant improvement (repeated measures ANOVA, $p=0.02$ ) in the $\mathrm{Hb}$ (mean of 118.10 (7.7) vs. $114.70(7.0) \mathrm{g} / \mathrm{l}$ in the control). However, as shows in figure 1 , a difference in mean change was not shown (mean (SEM) change of 5.0 (1.1) g/L in intervention group and $+2.4(1.3) \mathrm{g} / \mathrm{l}$ of control group, $p=0.14)$. The prevalence of anaemia dropped from $37 \%$ to $15 \%$ in the intervention group ( $p=0.03)$. The control group too had a drop from $44 \%$ to $26 \%$, but it did not reach a significant level (Figure 2, $p=0.43$ ). There was a significant difference $(p=0.05)$ in baseline SF status between groups. After correcting for the difference, a significant improvement $(p<0.001)$ was shown by the intervention group (Table 1$)$. Mean change in SF was +40.71 (3.97) $\mu \mathrm{g} / 1$ in the intervention group whereas control group had only +15.49 (3.54) $\mu \mathrm{g} / 1$ gain during this period (Figure $1, p<0.001$ ). Baseline iron deficiency prevalence $(4 \%)$ in the intervention group became zero percent after the intervention. Similarly the control group too showed a drop in the prevalence of iron deficiency from $14 \%$ to $7 \%$ $(p<0.05)$ (Figure 3$)$. As a surrogate marker of iodine status we, measured the free T4 level of these children. No difference in hormone levels was seen after correcting for baseline differences. No treatment effect was evident with regard to serum folate as well. 'Thriposha' supplement containing $450 \mathrm{mg} / \mathrm{meal}$ of calcium did not result in a significant improvement in the intervention group after correcting for baseline differences. Similarly serum zinc levels too did not show any significant improvement after correcting the baseline difference (Table 1). However, the mean serum zinc in the intervention group $(+0.67(0.17)$ $\mu \mathrm{mol} / 1$ ) improved significantly (Figure $1, p=0.008$ ). Over $60 \%$ of deficiency status (serum zinc $<9.945 \mu \mathrm{mol} / \mathrm{l}$ ) 
remained unaffected in both groups after the intervention. 'Thriposha' supplementation significantly improved the ceruloplasmin level to 434.06 (1.23) $\mathrm{mg} / \mathrm{l}$ in the intervention group ( $p<0.001)$. Further, the intervention group had mean change of $+117.11 \mathrm{mg} / \mathrm{l}$ whereas the control group had a change of $-16.10(5.58) \mathrm{mg} / \mathrm{l}(p<0.001)$ during this period (Figure 1). The mean change of serum retinol level observed in the interventional group $(+0.57(0.06) \mu \mathrm{mol} / \mathrm{l}$ was better than that was seen in the control group $(+0.36(0.09) \mu \mathrm{mol} / \mathrm{l}$, $p=0.05$ ) (Figure 1). There was no significant treatment effect ( $p=0.28$ ) seen after the intervention when corrected for the baseline differences of serum vitamin D levels among children. The intervention group had a mean change of $+32.18(5.72) \mathrm{nmol} / \mathrm{l}$ at the end of intervention, whereas control group had $-9.21(5.06) \mathrm{nmol} / \mathrm{l}(p<0.001)$ (Figure 1).

Table 1. Effect of 'Thriposha' supplementation on haematological and biochemical parameters ${ }^{1}$

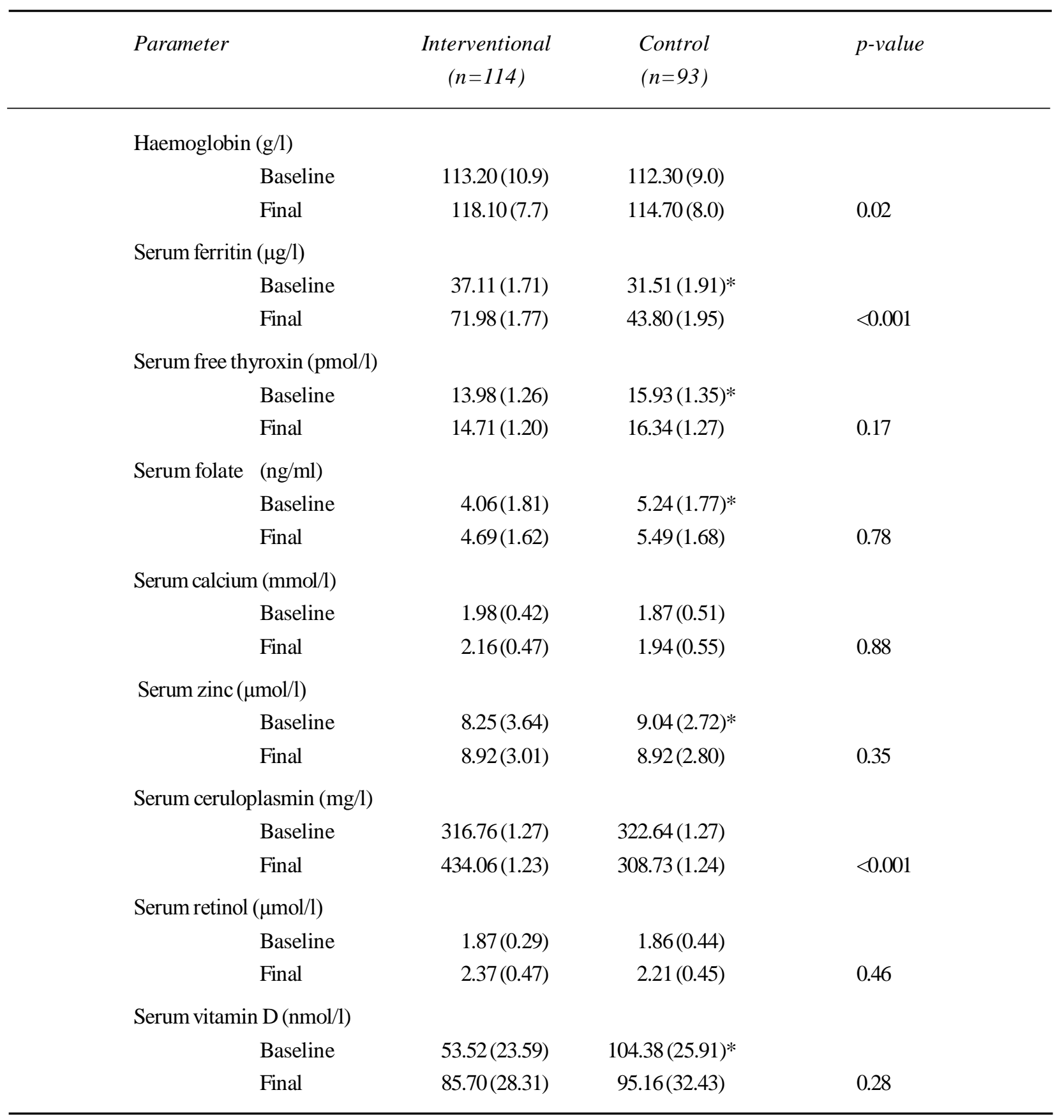

${ }^{1}$ Results presented as mean (SD) except for serum ferritin geometric mean (variance); $p$-value from the repeated measures ANOVA

* Indicates significant differences $(p<0.05)$ at the baseline 


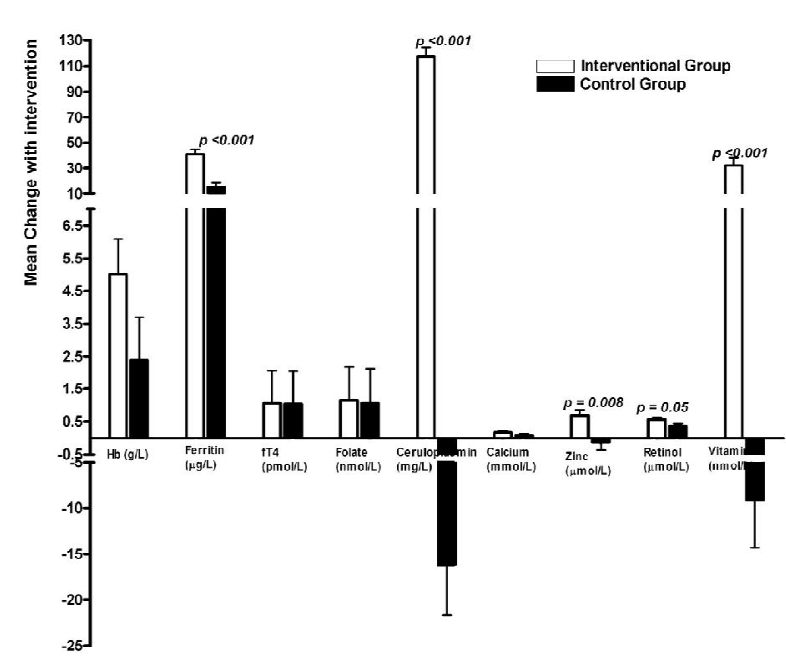

Figure 1. Mean change of haemoglobin and other trace minerals after the intervention.

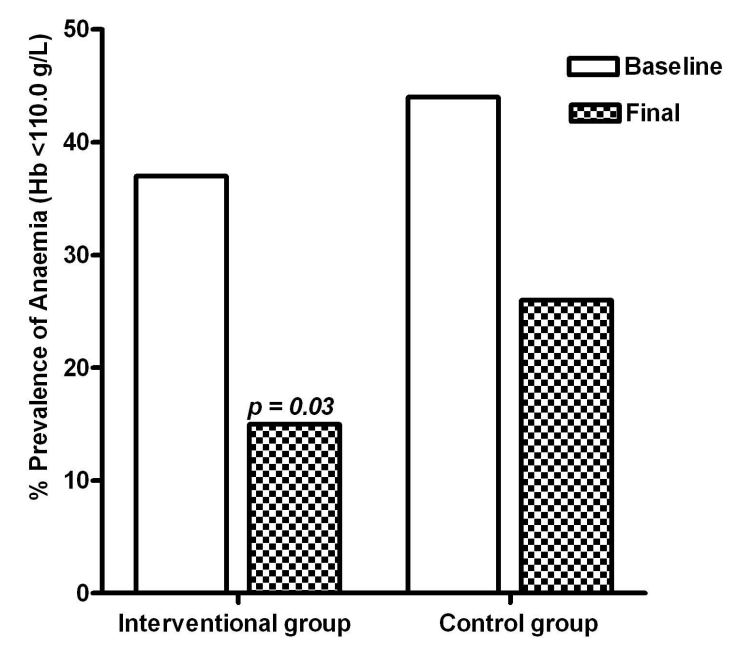

Figure 2. Effect of 'Thriposha' supplementation on anaemia $(\mathrm{Hb}<110.0 \mathrm{~g} / \mathrm{l})$ prevalence.

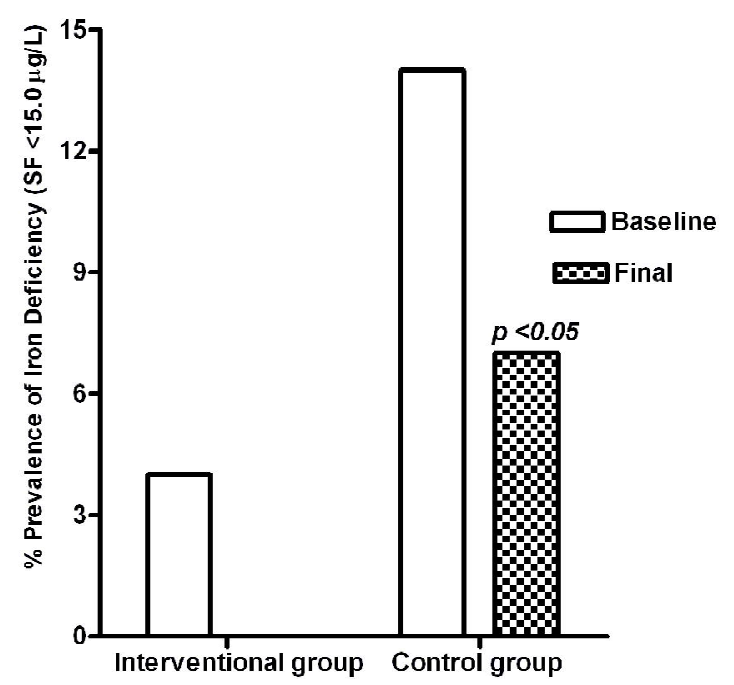

Figure 3. Effect of 'Thriposha' supplementation on serum ferritin deficiency.

\section{Discussion}

In this study, we examined the effects of a multinutrient fortified ready-to-eat cereal based food supplement on haematological and biochemical status of young children in Galle. Major study outcomes were improved $\mathrm{Hb}, \mathrm{SF}$ and ceruloplasmin levels and marginal effects on zinc, retinol and vitamin D status. The significant improvement in SF in the intervention group indicates a positive change in underlying iron status. As noted above, the prevalence of anaemia decreased in both groups, but the drop was significant only in the intervention group. Notwithstanding these significant improvements in iron status and reduction of anaemia, we did not observe complete recovery of $\mathrm{Hb}$ or prevention of anaemia. This could be the result of multiple factors, including incomplete recovery from deficiencies of iron, vitamin A, folate, vitamin B-12, and other nutrients necessary for optimal haematopoiesis. Other investigators have observed a lack of $\mathrm{Hb}$ response to iron supplements with and without micronutrients despite improved iron status and reduction of iron deficiency [3, 4].

Low levels of serum folate, calcium, ceruloplasmin and zinc levels were relatively common at the start of the study. However, by the end the deficiencies of anaemia, iron, calcium and ceruloplasmin were much less in the intervention group than in the control group. Children in the intervention group had a significant gain in serum zinc and retinol levels though the rate of deficiency did not change significantly. The total amount of zinc and retinol might have been inadequate to lower the deficiency levels in the intervention group. Serum ferritin, ceruloplasmin and serum retinol can be falsely elevated because of infection or inflammatory conditions. Although we did not measure acute phase proteins, we can expect that the influence of infection would be randomly distributed across the two groups on the basis of our study design.

Differences observed at the end of the study suggest a beneficial effect on zinc status derived from consumption of the 'Thriposha'. The high zinc: iron ratio (1:6) may have affected zinc absorption [5]. The lower zinc content (1.5 mg or one third of daily recommended allowance) may have resulted in lack of significant treatment effect in the intervention group. Our study was significantly limited by the inclusion of only one Health Division. Although our results show the benefits of 'Thriposha', a randomised, placebo controlled, double-blind study in multiple geographical areas over an extended period would be necessary to assess the efficacy of this programme at a national level.

In conclusion, this study demonstrated that regular consumption of 'Thriposha' compared with a control (CSB), for 9 months led to improvements in $\mathrm{Hb}$, reducing anaemia and several measures of micronutrient deficiency states in peri-urban preschool children in Galle. 


\section{Acknowledgments}

This study was done with financial assistance from the International Atomic Energy Agency (IAEA-SRL11958). Conventional 'Thriposha' and the CSB were received free of charge, through the Ministry of Health, Sri Lanka. Analysis of serum zinc and retinol were funded by a World Bank grant through the Nutrition Co-ordination Division, Ministry of Health.

\section{References}

1. van Stuijvenberg ME, Dhansay MA, Smuts CM. Longterm evaluation of a micronutrient-fortified biscuit used for addressing micronutrient deficiencies in primary school children. Public Health Nutrition 2001; 4: 1201- 9.

2. Anderson AJB. Interpreting Data: A first course in statistics. 8th edition. Chapman and Hall, UK, 1989.

3. Dallman PR, Looker AC, Johnson CL. Influence of age on laboratory criteria for the diagnosis of iron deficiency anaemia and iron deficiency in infants and children. In: Iron Nutrition in Health and Disease Hallberg L, Asp NG (eds.), John Libbey \& Company, London. 1996; 65-74.

4. Palupi L, Shultink W, Achadi E, Gross R. Effective community intervention to improve haemoglobin status in preschoolers receiving once-weekly iron supplementation. American Journal of Clinical Nutrition 1997; 65: 1057-61.

5. Allen L, Rosado J, Casterline J. Lack of hemoglobin response to iron supplementation in anaemic preschoolers with multiple micronutrient deficiencies. American Journal of Clinical Nutrition 2000; 71: 1485-94.

6. Peres JM, Bureau F, Neuville D. Inhibition of zinc absorption by iron depends on the ratio. Journal of Trace Element Biology 2001; 15: 237-41.

7. Hettiarachchi M, Liyanage C, Hilmers D. Changing the zinc:iron ratio in a cereal-based nutritional supplement has no effect on percent absorption of iron and zinc in Sri Lankan children. British Journal of Nutrition 2009; 1-8 (published online).

8. Rivera JA, Ruel MT, Santizo MC, Lonnerdal B, Brown $\mathrm{KH}$. Zinc supplementation improves the growth of stunted rural Guatemalan infants. Journal of Nutrition 1998; 128: 556-62. 